\title{
Criterion of vehicle stability in floodwaters based on theoretical and experimental studies
}

\author{
Junqiang Xia ${ }^{1}$, Roger A. Falconer ${ }^{2}$, Xuanwei Xiao ${ }^{1}$ and Caiwen Shu ${ }^{1}$ \\ (' ${ }^{1}$ tate Key Laboratory of Water Resources and Hydropower Engineering Science, Wuhan University, Wuhan 430072, China) \\ ( ${ }^{2}$ Hydro-environmental Research Centre, School of Engineering, Cardiff University, Cardiff, CF24 3AA, UK)
}

\begin{abstract}
Vehicles parked on streets or roads can cause various hazards to people and property when they are swept away by urban floods. It is therefore approproriate to investigate the criterion of vehicle stability for such flood conditions, especially for different scenarios and where the criterion for vehicle stability is usually represented by the incipient velocity for the vehicle. In the current study, different forces acting on a partially submerged vehicle are outlined, together with the corresponding expressions of these forces, and a mechanics-based formula of incipient velocity is given for partially submerged vehicles under different orientation angles. About 200 runs of flume experiments were conducted to obtain the conditions of water depth and corresponding velocity at the threshold of vehicle instability for three orientation angles, using two types of die-cast model vehicles at two model scales. Experimental data obtained from the large-scale model vehicles were then used to determine two parameters in the derived formula. Finally, incipient velocities for three vehicle orientation angles were estimated using two different approaches, including predictions for the scale ratios from the small-scale model vehicles and computations based on derived formula using the prototype vehicle parameters. These critical conditions for the prototype conditions, based on the scale ratios, compared well with the calculations obtained using derived formula, which guaranteed the predicative accuracy of the formula. In addition, the effect of different ground slopes on the vehicle incipient motion was also investigated, using similar experiments and based on the theory of similarity, which indicated that the incipient velocity for a small passenger vehicle on a ground slope of 1:50 was about 25\% lower than the value on flat ground for an incoming depth of $0.25 \mathrm{~m}$.
\end{abstract}

Keyword: Vehicle stability; Incipient velocity; Theoretical analysis, Flume experiments, Scale ratio; Similarity theory; Urban floods

\section{INTRODUCTION}

The probability of urban flooding has increased in recent years as a result of the global change in climatic and meteorological conditions. The risk of vehicles becoming unstable during urban flood events has therefore increased noticeably. Vehicles in urban areas usually tend to be unstable by sliding or floating during flood events, which further leads to various hazards, including causing injuries or mortality to passengers and pedestrians, damage to buildings, and even exacerbation of a 
flood event by blocking local hydraulic structures, such as culverts or bridges etc. Experience from past floods, particularly in the United States, shows that a common hazard that causes a risk of death or serious injury to people is due to the instability of vehicles in floodwaters (Defra and EA, 2006). Therefore, the stability of vehicles during urban flood events is a topic that has aroused recent interest from the Environment Agency in the UK and other flood management authorities around the world.

On 16 August 2004, the small town of Boscastle in the UK was struck by a devastating flash flood, and the Environment Agency reported that millions of pounds of damage was done to buildings, and more than 100 vehicles were swept away into the sea. Moreover, some vehicles and other large-size debris were caught under a local bridge, throttling the flow and causing the bridge to collapse, which caused a mass of floodwater to build up behind the bridge, thereby increasing flood risk in the streets of the town of Boscastle (EA, 2004).

On 29 August 2009, a small county of Chongqing city in China was struck by a large flash flood, and five vehicles parked on the floodplain were swept into the Yangtze River during the flood. These occurrences have highlighted the need for further investigation of the stability criterion of vehicles under various scenarios, from the viewpoint of flood risk management of urban areas.

Various criteria of vehicle stability in floodwaters have been studied widely in the form of incipient velocity, and existing stability criteria are based on the experimental studies and analytical results, conducted by e.g. Gordon and Stone (1973), Keller and Mitsch (1993), Xia et al. (2011) and Shu et al. (2011). Keller and Mitsch (1993) provided a simple method for estimating the forces exerted on a stationary vehicle in floodwaters. The method considered the vehicle mass and dimensions, buoyancy and drag forces, which were used to determine the incipient velocity of the vehicle. Xia et al. (2011) derived a formula to predict the incipient velocity for flooded vehicles, using the mechanical condition of sliding equilibrium, where more attention was focused on fully-submerged vehicles. Experimental data obtained for small-scale model vehicles were used to determine parameters in the derived formula. Subsequently, Shu et al. (2011) conducted a similar procedure to derive a formula for the incipient velocity for partially submerged vehicles, and the derived formula was validated using experimental data based on the principles of similarity and scale ratios. It should be noted that existing studies seldom account for the effects of vehicle orientation angles and ground slope on the threshold of vehicle instability. However, vehicles parked on roads or streets could be swept away under various scenarios, including different orientation angles and ground slopes. Teo et al. (2012) undertook extensive research into the criteria for vehicle stability, by conducting a series of scaled model experiments in two laboratory hydraulics flumes, for different vehicle orientation angles and ground slopes. However, the principles of 
similarity were not considered in these experiments, so that the obtained experimental results using the model vehicles could not be applied directly to the corresponding prototype vehicles.

Shand et al. (2011) presented a comprehensive review of existing safety criteria for flooded vehicles, where they proposed interim criteria for stationary stability of three types of common vehicles. As mentioned in this report, substantial changes in vehicle design have occurred over the past decades, especially in vehicle planform area, vehicle weight and ground clearance. Recent urban floods in the UK and China have indicated that vehicles in urban areas are generally swept away under various conditions, including different vehicle orientation angles and ground slopes. Therefore, it was thought appropriate to investigate the stability criteria for partially submerged vehicles in urban areas and for various scenarios.

In this study the stability criterion for partially submerged vehicles, for various scenarios, was investigated herein. Various forces exerted on stationary vehicles in floodwaters are outlined, together with the corresponding expressions for these forces. A mechanics based formula of incipient velocity is given for partially submerged vehicles, assuming that the inside space of a vehicle can not be filled quickly by the flood water. About 200 runs of flume experiments were conducted under three vehicle orientation angles and for three ground slopes, using two types of die-cast vehicles. The similarity principles of shape, motion and forces were strictly followed. The parameters in the derived formula were calibrated using experimental data obtained for larger scale model vehicles. The critical motion conditions for prototype vehicles were estimated both using the model scale ratios and using the derived formula. In addition, the effect of various ground slopes on the threshold of vehicle instability was also investigated using the experiments.

\section{Derivation of incipient velocity formula}

In this section the various forces exerted on a stationary vehicle in floodwater are considered, and the corresponding equations obtained using a mechanics based approach to establish the incipient velocity for a partially submerged vehicle, based on the equilibrium of sliding. More details of the force analysis are given in Xia et al. (2011) and Shu et al. (2011).

\subsection{Force analysis of a stationary vehicle in floodwater}

As the floodwater flows around a partially-submerged vehicle parked on a street or road, the vehicle usually experiences four forces, including the drag force $\left(F_{D}\right)$, the effective weight $\left(F_{G}\right)$, the normal reaction force from the ground $\left(F_{N}\right)$, and the frictional force between the tyre and ground surface $\left(F_{R}\right)$, as shown in Fig. 1. In this study, two key assumptions are included in the force analysis. The first is that the wheels of a vehicle are all locked against any motion as it is parked on a street or road (i.e. the brakes are on), and thus $F_{R}$ is produced to resist the vehicle from sliding along the ground surface. The second is that the vehicle is regularly maintained and has enough 
sealing capacity so that its inside space is assumed not to fill with floodwater at the initial stage of a flood event, even if the water level outside the vehicle is higher than the height of ground clearance. Therefore, the second assumption adopted in this study is different from previous research reported by some of the authors (Xia et al., 2011).

The vehicle dimensions are characterized by the length $l_{c}$, width $b_{c}$, and roof height $h_{c}$. Under the situation of partial submerge, $F_{G}$ can be expressed in the form as

$$
F_{G}=a_{c} l_{c} b_{c}\left(h_{c} \gamma_{c}-R_{f} h_{f} \gamma_{f}\right)
$$

where $\gamma_{c}$ and $\gamma_{f}$ are the specific weights of the vehicle and water, respectively; $\alpha_{c}$ is a coefficient representing the proportion of effective vehicle volume; $h_{f}$ is the incoming depth; and $R_{f}=$ $h_{c} \gamma_{c} /\left(h_{k} \gamma_{f}\right)$, in which $h_{k}$ is the critical water depth at which the vehicle starts to float.

The drag force acting on the side of the vehicle is given generally as (Chien and Wan, 1999)

$$
F_{D}=C_{d} A_{d} \gamma_{f} \cdot\left(u_{b}\right)^{2} /(2 g)
$$

where $C_{d}$ is the drag coefficient; $u_{b}$ is the representative near-bed velocity; $g$ is the gravitational acceleration; and $A_{d}$ is the submerged area projected normal to the incoming flow, with $A_{d}=$ $\alpha_{d}\left(h_{f} \cdot b_{c}\right)$ for the case of inflow direction parallel to the vehicle length. The empirical parameter $a_{d}$ relates only to the detailed shape of a vehicle side projected normal to the incoming flow, as the incoming depth is greater than the chassis height. For a specified vehicle category, $C_{d}$ is evidently a function of the Reynolds number. In this study, it is assumed that $C_{d}$ is a constant for large values of Reynolds number, and has the same magnitude in the model and prototype (Chanson, 2004). A comprehensive parameter including $C_{d}$ and other coefficients is introduced in the current analysis.

The frictional force is thought to be equal to the total resistance acting on the four wheels by the ground surface, as all of the wheels of a parked vehicle are assumed to be locked against sliding. This force can be expressed by $F_{R}=\mu F_{N}$, in which $\mu$ is the friction coefficient between the tyre and wet ground surface. If the vehicle is in equilibrium of stationary sliding, then $F_{N}=F_{G}$. Therefore, $F_{R}$ can be expressed in the form

$$
F_{R}=\mu F_{N}=\mu F_{G}=\mu\left[a_{c} l_{c} b_{c}\left(h_{c} \gamma_{c}-R_{f} h_{f} \gamma_{f}\right)\right]
$$

There are many factors influencing the coefficient of friction, covering the tyre condition and the road surface condition (Cox and Ball, 2011). Therefore, $\mu$ is a key parameter in determining the critical velocity of a flooded vehicle, which is also included in the derived formula as a comprehensive parameter.

\subsection{Establishment of incipient velocity formula}

Vehicles parking on streets or roads can start to move in the form of sliding or floating under the action of the floodwater, depending upon the conditions of the incoming flow. At the initial stage of flooding, vehicles often move mainly in the form of sliding, especially in urban floods with low depth and high velocity. As the vehicle starts to slide along the ground surface, the stationary 
frictional force preventing the vehicle from sliding is just balanced by the drag force induced by the incoming flow, so that the corresponding criterion of instability threshold can be written as $F_{D}=F_{R}$. Substitution of Eqs. (2) and (3) into this expression yields

$$
u_{b}=\sqrt{\mu a_{c} /\left(a_{d} C_{d}\right)} \sqrt{2 g l_{c}\left[h_{c} \rho_{c} /\left(h_{f} \rho_{f}\right)-R_{f}\right]}
$$

where $\rho_{c}$ and $\rho_{f}$ are the densities of the vehicle and water, respectively. In general, $u_{b}$ can not be readily determined, and the depth-averaged velocity $U$ is then used in its place. The vertical distribution of velocity is expressed as $u=(1+\beta) U\left(y / h_{f}\right)^{\beta}$, in which $\beta$ is a coefficient obtained from calibration; $y$ is the vertical distance from the bed; and $u$ is the velocity at elevation $y$ (Zhang and Xie, 1993; $\mathrm{Wu}, 2007)$. Assuming that the representative height for $u_{b}$ is $\alpha_{b} h_{c}$, then $u_{b}=$ $(1+\beta) U\left(\alpha_{b} h_{c} / h_{f}\right)^{\beta}$, in which $\alpha_{b}$ is a coefficient. Substituting the expression of $u_{b}$ into Eq. (4), the incipient velocity $U_{c}$ for partially-submerged vehicles in line (or parallel) with the inflow direction is given as

$$
U_{c}=\alpha\left(h_{f} / h_{c}\right)^{\beta} \sqrt{2 g l_{c}\left[\rho_{c} h_{c} /\left(\rho_{f} h_{f}\right)-R_{f}\right]}
$$

where $\alpha=\left[\left(\mu / C_{d}\right)\left(\alpha_{c} / \alpha_{d}\right)\right]^{0.5}\left[(1+\beta)\left(\alpha_{b}\right)^{\beta}\right]^{-1}$; both $\alpha$ and $\beta$ are related to vehicle shape, tyre type and roughness of road surface, which are determined through the following experiments. For the case where the inflow direction is perpendicular to the side of a vehicle, then Eq.(5-1) needs to be rewritten as:

$$
U_{c}=\alpha\left(h_{f} / h_{c}\right)^{\beta} \sqrt{2 g b_{c}\left[\rho_{c} h_{c} /\left(\rho_{f} h_{f}\right)-R_{f}\right]}
$$

It should be noted that the magnitude of the friction coefficient $\mu$ in Eq.(5-1) could be different from that in Eq.(5-2), and the following tests for the friction coefficient indicate that the value of $\mu$ for Eq.(5-1) is usually less than the value for Eq.(5-2).

\section{Experiments based on the theory of similarity}

\subsection{Description of the test flume}

A series of tests have been conducted in a flume of the Experimental Hall for Sediment and Flood Control Engineering, Wuhan University, China, to investigate the conditions of incipient motion for partially-submerged vehicles under three vehicle orientation angles. The horizontal flume is $60 \mathrm{~m}$ long, $1.2 \mathrm{~m}$ wide, and with the bed covered by a thin cement layer and two glass sides. To determine the threshold of vehicle instability, the discharge in the flume was adjusted gradually to achieve a steady state, and the incoming depth and corresponding depth-averaged velocity were recorded when the flooded vehicle started to slide. To estimate the critical conditions for prototype vehicles using the experimental results and the corresponding scale ratios, strict similarity principles were followed to design the flume tests. The data obtained have been used to calibrate the two key parameters $\alpha$ and $\beta$ in Eq. (5). 


\subsection{Design of flume experiments}

A physical model is a scaled representation of a hydraulic flow situation, and the flow conditions are assumed to be similar to those in the prototype if the physical model follows the similarity of form, motion and forces (Zhang and Xie, 1993; Chanson, 1999). The size of the model vehicles were required to strictly follow geometric similarity, and two typical die-cast model vehicles were selected in this study. The physical models provide a good comparison on how the vehicle's size, design shape and weight can affect the threshold of vehicle instability in floodwaters. Two sets of different model scales were adopted, with a large die-cast model scale of 1:14 and with a smaller scale of 1:24. Detailed vehicle specifications are given in Table 1.

The tests were designed in an undistorted scale model, due to the use of vivid die-cast models, with the scale ratio of length $\lambda_{L}$ being equal to that of height $\lambda_{H}$, namely $\lambda_{L}=\lambda_{H}=14$ or 24 . According to requirements for kinematic similarity, the scale ratio of the inertia force to gravity gives the relationship between the scale ratios of velocity $\lambda_{U}$ and length $\lambda_{L}$, which can be expressed as $\lambda_{U}=$ $\left(\lambda_{L}\right)^{0.5}$. Dynamic similarity implies that the ratios of the prototype forces to model forces are equal to the same scale ratio of $\lambda_{F}$, which is also equivalent to $\left(\lambda_{L}\right)^{3}$. Herein, the selected density of a model vehicle was nearly equal to that of the corresponding prototype, so that $\lambda_{F_{G}}=\lambda_{F}$ was also guaranteed. With the relatively high values of the Reynolds number in the flume tests, the drag coefficient was considered constant for a specified shape (Chanson,2004), so that $C_{d}$ for the model was nearly equal to that for the prototype. Therefore, the similarity principle of drag coefficient was guaranteed for each model and prototype vehicle used, resulting in $\lambda_{F_{D}}=\lambda_{F}$. The friction coefficients for the two model vehicles were measured in the flume, and the mean friction coefficient measured was 0.25 for the case where the inflow direction was parallel to the vehicle length, and 0.75 for the case where the inflow direction was perpendicular to the vehicle length (i.e. where the flow was normal to the vehicle side). Therefore, the range of friction coefficients for the model vehicles corresponded well with the prototype range (Gerard, 2006). It was concluded that the friction coefficient for the model was nearly equal to that for the prototype, so that $\lambda_{F_{R}}=\lambda_{F}$.

Two model vehicles used in this study including a Honda Accord (i.e. a small passenger vehicle) and an Audi Q7 (a 4 wheel drive saloon vehicle). It should be noted that the density of a model vehicle is almost equal to that of a prototype one. A prototype vehicle would float when the water depth around the vehicle exceeded a critical depth if the inside space did not be fill quickly with floodwater. These critical floating depths ranged from 0.45 to $0.67 \mathrm{~m}$ for the prototype vehicles. For each test the wheels of the vehicle were locked, and therefore the incipient motion under sliding only was considered herein. To effectively prevent water from seeping into the inside space of a vehicle, its interior space was filled with light foam, to ensure $\lambda_{\rho_{c}}=1$.

\subsection{Analysis of experimental data}


Three vehicle orientation angles were considered in the study, as shown in Fig. 2. The angle of $0^{\circ}$ or $180^{\circ}$ meant that the vehicle rear or front was facing the incoming flow, while an angle of $90^{\circ}$ meant that the lateral side of a vehicle was facing the inflow direction. The test results indicated that there was not a substantial difference in the conditions of incipient motion for the orientation angles of $0^{\circ}$ and $180^{\circ}$, because the submerged area projected normal to the incoming flow for the former was virtually equivalent to that for the latter for a partially-submerged vehicle. However, the test results for the case of $90^{\circ}$ were evidently different from those for the case of $0^{\circ}$ or $180^{\circ}$. Therefore, the following analysis does not consider the difference in the test results for the cases of $0^{\circ}$ and $180^{\circ}$, and the test results for the case of $90^{\circ}$ is considered separately.

Fig. 3 shows the depth-incipient velocity relationships for the two vehicle types, for different orientation angles. From the experimental data it can be observed that: (i) the incipient velocity increases with a decrease in the incoming depth for each vehicle and any orientation angle; (ii) similar relationships between $h_{f}$ and $U_{c}$ are obtained for the angles of $0^{\circ}$ and $180^{\circ}$; (iii) for the same velocity, the critical depth at which the Audi starts to float is greater than that for the Honda, as shown in Fig.3a or 3c; and (iv) for the same depth, the incipient velocity for $90^{\circ}$ is less than that for $0^{\circ}$ or $180^{\circ}$, which applies to both model vehicles, i.e. the model Honda and Audi.

\subsection{Parameter determination}

For the case of $0^{\circ}$ and $180^{\circ}$ orientations, Eq. (5-1) is transformed to $U_{c} /\left[2 g l_{c}\left(R_{\rho} R_{h}-R_{f}\right)\right]^{0.5}=$ $\alpha\left(h_{f} / h_{c}\right)^{\beta}$ in order to determine the parameters of $\alpha$ and $\beta$, in which $R_{\rho}=\rho_{c} / \rho_{f}$ and $R_{h}=h_{c} / h_{f}$. For a given vehicle type, the values of $l_{c}, h_{c}, \rho_{c}, \rho_{f}$ and $R_{f}$ are constant. Therefore, both $\alpha$ and $\beta$ result from the method of logarithmic transformation and linear regression (Xia et al., 2011), as stated in Table 2. For the case of a $90^{\circ}$ orientation, then the parameters $\alpha$ and $\beta$ in Eq. (5-2) can also be calibrated using the corresponding experimental data. It can be seen from Table 1 that the values of $\alpha$ range from 0.2 and 0.5 and the values of $\beta$ vary from -0.2 to -0.6 , for different vehicles and under various orientation angles. $\beta<0$ for each partially-submerged vehicle indicates that the value of $U_{c}$ increases as the incoming depth decreases.

\section{Validation of the formula}

\subsection{Two approaches to estimating incipient velocity}

Incipient velocities for partially-submerged prototype vehicles in floodwaters can be estimated using two different approaches, including: (i) estimation of the velocity using the scale ratios from the small-scale model vehicles, and (ii) computation of the velocity based on the derived formula using the parameters for the prototype. The determination process of parameters in Eq. (5) adopted the experimental data from the large-scale model vehicles, and therefore it was thought that the first approach, using the experimental data from the small-scale model vehicles, could be used to independently validate the predictive accuracy of the derived formula. 
Since these model tests strictly followed the similarity principles, and the incipient velocities measured were for a specified water depth and for a particular model vehicle, the velocities could be directly used to estimate the incipient motion conditions for the corresponding prototype vehicle, according to the scale ratios of depth and velocity. These scaling relationships are written as

$$
\lambda_{h_{f}}=h_{f p} / h_{f m}=\lambda_{L} \text { and } \lambda_{U_{c}}=U_{c p} / U_{c m}=\sqrt{\lambda_{L}}
$$

where subscripts $p$ and $m$ refer to prototype and model parameters, respectively. Therefore, Eq. (6) can be re-written as

$$
h_{f p}=h_{f m} \times \lambda_{L} \text { and } U_{c p}=U_{c m} \times \sqrt{\lambda_{L}}
$$

where $\lambda_{L}=24$ for the small-scale model vehicles in the formula validation process. Substitution of the prototype parameters into Eq. (5-1) or Eq. (5-2) enables the critical velocity to be obtained for a specified depth, for each prototype vehicle, using the corresponding values of $\alpha$ and $\beta$ in Table 2.

\subsection{Formula validation}

The critical conditions obtained using Eq. (7) for the small-scale model vehicles are in close agreement with the calculations from the derived formula of Eqs. (5-1) and (5-2), as shown in Fig. 4, which confirms the accuracy of the critical conditions for partially submerged prototype vehicles, estimated using these two approaches. The results from Fig. 4 also show that:

(i) with an increase in the incoming depth, then the corresponding incipient velocity for each vehicle decreases, mainly due to an increase in the projected area normal to the inflow direction and a decrease in the frictional force. For the Honda model and orientations of $0^{\circ}$ and $180^{\circ}$, the magnitude of the incipient velocity was about $3.0 \mathrm{~m} / \mathrm{s}$ for an incoming depth of $0.3 \mathrm{~m}$, while the velocity decreased to about $1.3 \mathrm{~m} / \mathrm{s}$ for $h_{f}=0.4 \mathrm{~m}$ (Fig. 4a);

(ii) for the same incoming depth, the incipient velocity for the orientations of $0^{\circ}$ and $180^{\circ}$ was slightly greater than for the $90^{\circ}$ orientation for Honda model, while the former was evidently higher than the latter for Audi model (Fig. 4b). For each vehicle under the same water depth, the drag force for the case of $90^{\circ}$ orientation was usually higher than that for $0^{\circ}$ or $180^{\circ}$ orientations due to the larger projected area, while the friction coefficient for the former was twice the value for the latter. Therefore, the composite effect of these two forces leads to the fact that the difference in the incipient velocities under different vehicle orientation angles is limited for the same vehicle;

(iii) the difference in the incipient velocities for different vehicle orientation angles decreased with an increase in the incoming depth. As the incoming depth approached the critical floating depth, this difference could be neglected, especially for the Honda model (Fig.4a);

(iv) the model Honda Accord was the easier vehicle to start sliding in floodwaters, relative to the Audi Q7 due to its lighter kerb weight and lower ground clearance height. For example, for the 
$0^{\circ}$ or $180^{\circ}$ orientations, the incipient velocity for $h_{f}=0.35 \mathrm{~m}$ for the Honda was about $2.0 \mathrm{~m} / \mathrm{s}$, which was much lower than the corresponding value for the Audi, which was $4.3 \mathrm{~m} / \mathrm{s}$.

\subsection{Effect of different ground slopes}

Vehicles in urban areas are frequently parked on streets or roads with different ground slopes, and hence it is appropriate to investigate the effect of different ground slopes on the incipient motion, for different vehicles. In this study, similar incipient motion experiments using the small-scale model vehicles were also conducted in the flume under two ground slopes of 1:100 and 1:50 for the orientation angle of $180^{\circ}$. These experiments also followed strictly the principles of similarity, and the obtained data were directly used to estimate the incipient motion conditions for the corresponding prototype vehicle, based on the scale ratios.

Fig. $5 a$ and $5 b$ indicate that the effect of different ground slopes on the incipient motion for the Honda and Audi model vehicles, respectively. It can be seen from Fig. 5 that the incipient velocities for the Honda were 3.9, 3.3 and $2.9 \mathrm{~m} / \mathrm{s}$, respectively, for the same incoming depth of $0.25 \mathrm{~m}$, for a flat slope and for slopes of 1:100 and 1:50, while the values of $U_{c}$ for the Audi were 4.4, 3.9 and 3.6 $\mathrm{m} / \mathrm{s}$, respectively, for a depth $h_{f}=0.35 \mathrm{~m}$. Therefore, it can be concluded from Fig. 5 that for the same incoming depth, the incipient velocity decreased with an increase in the ground slope. This can be explained based on the force analysis. When a vehicle is parked on flat ground in floodwater, the frictional force $\left(F_{R}\right)$ is equal to the product of the friction coefficient $(\mu)$ and the normal reaction from the ground $\left(F_{N}\right)$, which is equal to the effective weight of the vehicle $\left(F_{G}\right)$. When the ground slope is at an angle $\theta$, then the value of $F_{N}$ is reduced to $F_{G} \cos \theta$, with the value of $F_{R}$ decreasing to $\mu F_{G} \cos \theta$. At the same time, the driving force causing the vehicle to slip is increased to $F_{D}+F_{G} \sin \theta$. Therefore, the decrease in the restoring force and the increase in the driving force leads to a reduction in the incipient velocity on sloping ground, as compared with the value on flat ground.

\section{Conclusions}

Vehicles parked on flooded streets or roads can become unstable under various scenarios when they are swept away by urban floods, and can then cause hazards to people and property in urban areas.

The criterion of vehicle stability in floodwaters under different orientation angles and ground slopes was investigated based on the theoretical and experimental studies, and the conclusions obtained from the investigation include:

(i) Various forces acting on a partially submerged vehicle were outlined, and a mechanics based formula for the incipient velocity has been obtained. It was assumed that the inside space of a vehicle would not be filled quickly by the floodwater, and the vehicle could float for a relatively larger depth. Flume tests were conducted using two types of die-cast model vehicles, following similarity theory. 
The data obtained from large scale model vehicles were used to determine two parameters in the derived formula and for three orientation angles.

(ii) Two approaches were used to estimate the conditions of critical motion for partially submerged vehicles in floodwaters, including the predictions by the scale ratios from the small scale model vehicles and the computations obtained from the derived formulae using prototype parameters. The corresponding incipient velocities obtained using the former were in close agreement with the results obtained from the latter. These results therefore provide a preliminary quantified criterion of hazard level for vehicles parked in flood-prone urban areas for various orientation angles.

(iii) The effect of different ground slopes on the incipient motion was also investigated using similar experiments, with the results indicating that for the same incoming depth, the incipient velocity on sloping ground would be lower than the corresponding value on flat ground, for each vehicle type.

\section{Acknowledgements}

The work was partly supported by the 2012 Award for the Research Exchanges with China and India from The Royal Academy of Engineering, UK. It was also partly supported by the Program for New Century Excellent Talents in University from the Chinese Ministry of Education (NCET-10-0619), and by the Natural Science Foundation of China (Grant No. 51079103).

\section{References}

Chanson H (2004). The hydraulics of open channel flow: an introduction ( $2^{\text {nd }}$ ed). Elsevier Butterworth-Heinemann, Oxford UK. Chien N, Wan ZH (1999). Mechanics of sediment transport. ASCE Press.

Cox RJ and Ball EJ (2001). Stability and safety in flooded streets. In: Proceedings of conference on hydraulics in Civil Engineering. The Institution of Engineers, Barton Australia, pp. 239-248.

Defra and Environment Agency (EA) (2006). Flood and Coastal Defence R\&D Programme, R\&D outputs: Flood Risks to People (Phase 2 Project Record, FD2321/PR). <www.defra.gov.uk/environ/fcd/research>.

Environment Agency (2004). Living with the risk: the floods in Boscastle and North Cornwall on 16 August 2004. Environment Agency Report, Exeter UK.

Gerard M (2006). Tyre-road friction estimation using slip-based observers. Master thesis. Department of Automatic Control, Lund University, Lund Sweden.

Gordon AD, Stone PB (1973). Car stability on road floodways. Technical Report 73/12. University of New South Wales, Manly Vale, Australia.

Keller RJ, Mitsch B (1993). Safety aspects of design roadways as floodways. Research Report No. 69. Urban Water Research Association of Australia, Australia.

Shand TD, Cox RJ, Blacka MJ, Smith GP (2011). Appropriate safety criteria for vehicles: Literature Review (Stage 2). Report Number P10/S2/020. Australian Rainfall and Runoff (AR\&R), Manly Vale, Australia.

Shu CW, Xia JQ, Falconer RA and Lin BL (2011). Incipient Velocity for Partially Submerged Vehicles in Floodwaters. Journal of hydraulic research, 49(6): 709-717.

Teo FY, Xia JQ, Falconer RA and Lin BL (2012). Experimental studies on the interaction between vehicles and floodplain flows. International Journal of River Basin Management,10(2): 149-160.

Wu WM (2007). Computational river dynamics. Taylor \& Francis Group, London.

Xia JQ, Teo FY, Lin BL, Falconer RA (2011). Formula of incipient velocity for flooded vehicles. Natural Hazards, 58(1): 1-14.

Zhang RJ, Xie JH (1993). Sedimentation research in China. China Water and Power Press, Beijing. 


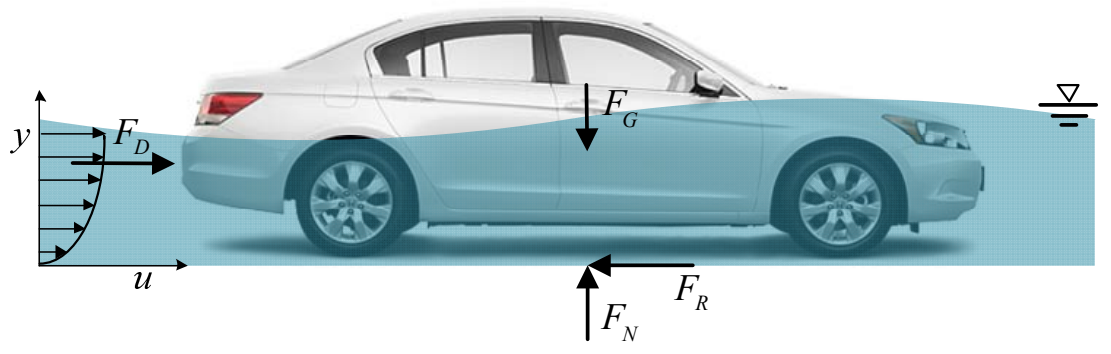

Fig.1 Schematic illustration of the key forces acting on a stationary vehicle 


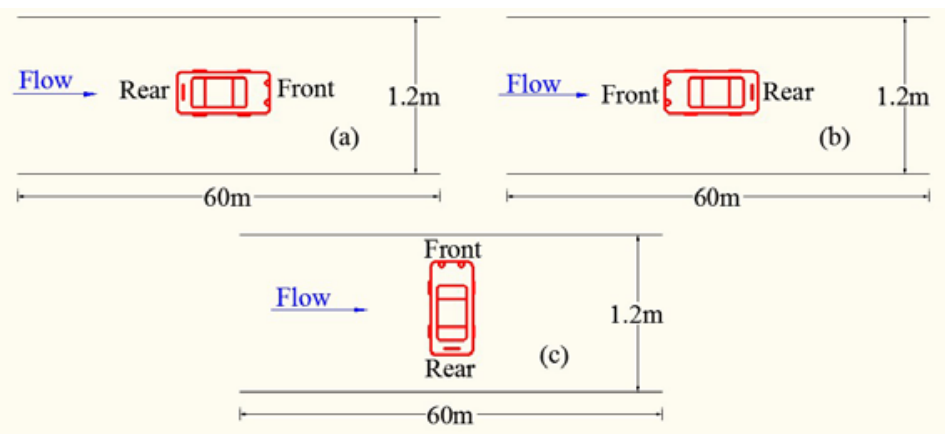

Fig.2 Sketch of vehicles for different orientation angles of: (a) $0^{\circ}$; (b) $180^{\circ}$ and (c) $90^{\circ}$
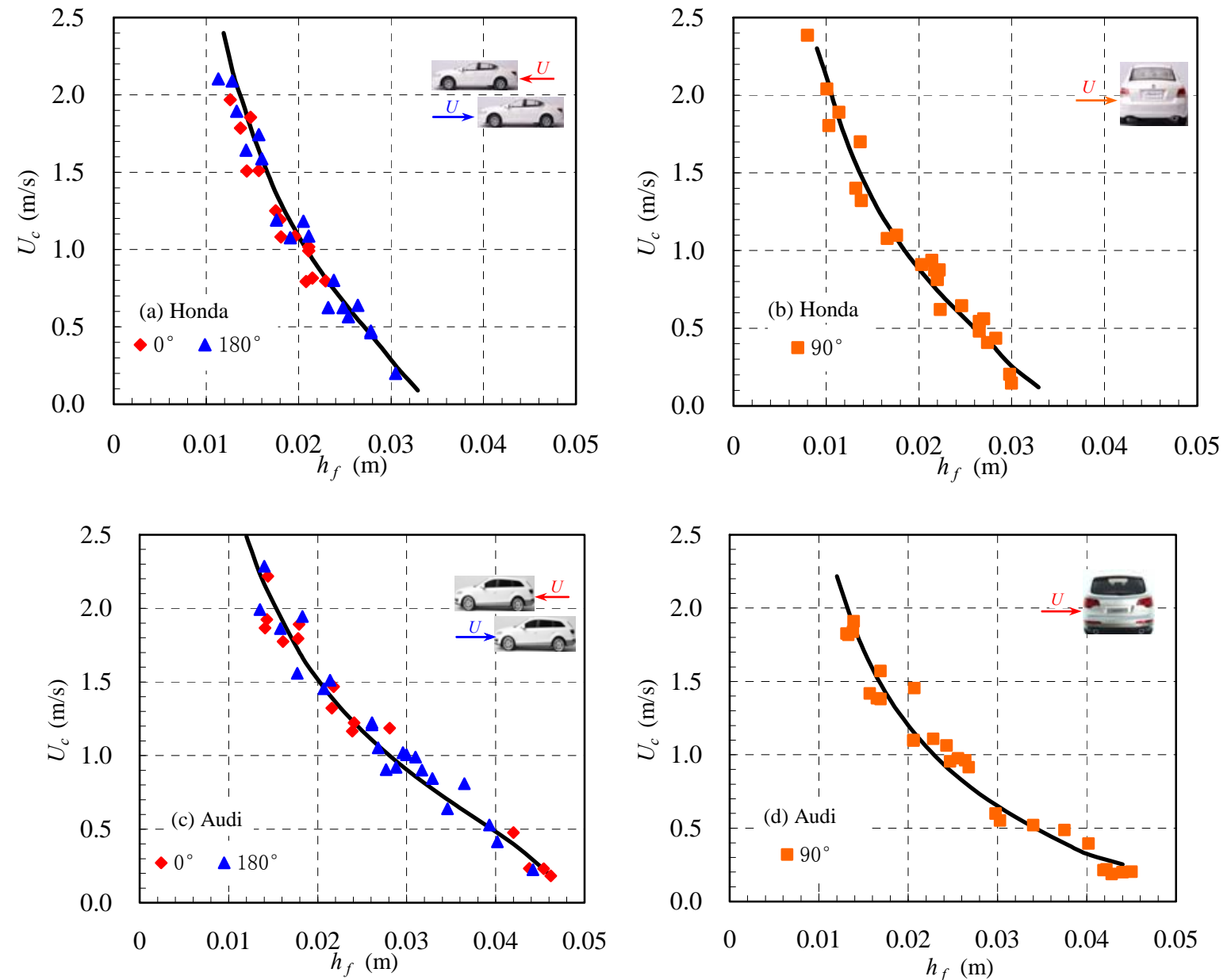

Fig. 3 Depth-incipient velocity relationships for large-scale model vehicles for different orientation angles 



Fig. 4 Comparison between estimated incipient velocity for prototype vehicles using two different approaches
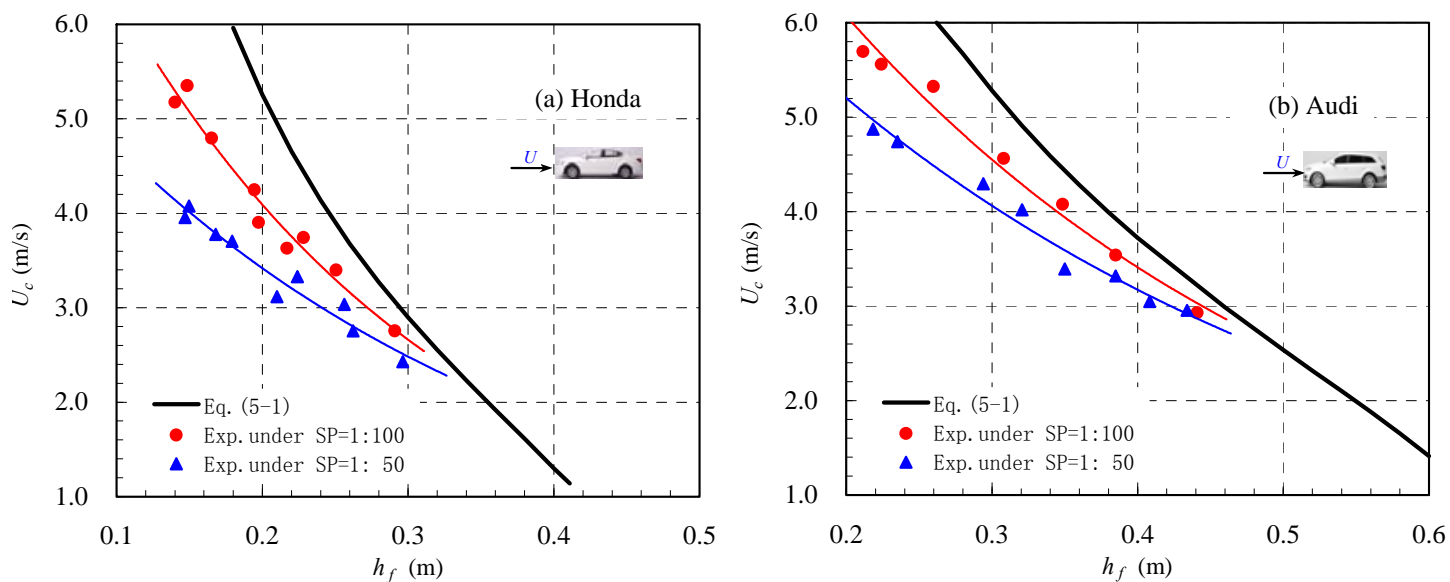

Fig. 5 Effect of different ground slopes on incipient velocity for Honda and Audi vehicles

Table 1 Specifications for model and prototype vehicles

\begin{tabular}{|c|c|c|c|c|c|}
\hline \multirow{2}{*}{ Vehicles } & Scale & Length (m) & Width (m) & $\begin{array}{c}\text { Height } \\
(\mathrm{m})\end{array}$ & $\begin{array}{c}\text { Kerb Weight } \\
(\mathrm{kg})\end{array}$ \\
\hline \multirow{2}{*}{$\begin{array}{c}\text { Honda } \\
\text { (Accord) }\end{array}$} & Prototype(1:1) & 4.945 & 1.845 & 1.480 & 1631 \\
\cline { 2 - 6 } & Model(1:14) & 0.353 & 0.134 & 0.107 & 0.597 \\
\cline { 2 - 6 } & Model(1:24) & 0.205 & 0.078 & 0.062 & 0.126 \\
\hline \multirow{2}{*}{$\begin{array}{c}\text { Audi } \\
\text { (Q7) }\end{array}$} & Prototype(1:1) & 5.089 & 1.983 & 1.737 & 2345 \\
\cline { 2 - 6 } & Model(1:14) & 0.365 & 0.140 & 0.123 & 0.855 \\
\cline { 2 - 6 } & Model(1:24) & 0.213 & 0.082 & 0.070 & 0.166 \\
\hline
\end{tabular}


Table 2 Parameters in the formula for incipient velocity

\begin{tabular}{|c|c|c|c|c|c|}
\hline \multirow{2}{*}{ Vehicles } & \multirow{2}{*}{$\begin{array}{c}\text { Orientation } \\
\text { angles }\end{array}$} & \multicolumn{4}{|c|}{ Parameters } \\
\cline { 3 - 4 } & & $\alpha$ & $\beta$ & $h_{k}(\mathrm{~m})$ & $R_{f}$ \\
\hline \multirow{2}{*}{$\begin{array}{c}\text { Honda } \\
\text { (Accord) }\end{array}$} & $0^{\circ}$ and $180^{\circ}$ & 0.212 & -0.562 & \multirow{2}{*}{0.45} & 0.650 \\
\cline { 2 - 4 } & $90^{\circ}$ & 0.492 & -0.344 & & \\
\hline \multirow{2}{*}{$\begin{array}{c}\text { Audi } \\
\text { (Q7) }\end{array}$} & $0^{\circ}$ and $180^{\circ}$ & 0.438 & -0.219 & \multirow{2}{*}{0.67} & 0.551 \\
\cline { 2 - 4 } & $90^{\circ}$ & 0.367 & -0.451 & & \\
\hline
\end{tabular}

